\title{
Multivariable polynomial injections on rational numbers
}

\author{
by
}

\author{
BJorn PoOnen (Cambridge, MA)
}

1. Introduction. Harvey Friedman asked whether there exists a polynomial $f(x, y) \in \mathbb{Q}[x, y]$ such that the induced map $\mathbb{Q} \times \mathbb{Q} \rightarrow \mathbb{Q}$ is injective. Heuristics suggest that most sufficiently complicated polynomials should do the trick. Don Zagier has speculated that a polynomial as simple as $x^{7}+3 y^{7}$ might already be an example. But it seems very difficult to prove that any polynomial works. Both Friedman's question and Zagier's speculation are at least a decade old (see [Cor99, Remarque 10]), but it seems that there has been essentially no progress on the question so far.

Our theorem gives a positive answer conditional on a small part of a well-known conjecture.

Theorem 1.1. Let $k$ be a number field. Suppose that there exists a homogeneous polynomial $F(x, y) \in k[x, y]$ such that the $k$-rational points on the surface $X$ in $\mathbb{P}^{3}$ defined by $F(x, y)=F(z, w)$ are not Zariski dense in $X$. Then there exists a polynomial $f(x, y) \in k[x, y]$ inducing an injection $k \times k \rightarrow k$.

REMARK 1.2. If $F(x, y)$ is separable (or equivalently, squarefree) and homogeneous of degree at least 5 , then $X$ is of general type. So the hypothesis in Theorem 1.1 would follow from the Bombieri-Lang conjecture that $k$-rational points on a surface of general type are never Zariski dense.

REMARK 1.3. As the proof of Theorem 1.1 will show, if we have an algorithm for determining the Zariski closure of the set of $k$-rational points on each curve or surface of general type, then we can construct $f(x, y)$ explicitly.

REMARK 1.4. To prove that a nonzero homogeneous polynomial $F(x, y)$ defines an injection $k \times k \rightarrow k$ is to prove that $X(k)$ is contained in the line $x-z=y-w=0$. If $F$ is separable, then $X$ is a smooth projective 
hypersurface in $\mathbb{P}^{3}$, so it is simply connected. But as far as we know, there is not a single simply connected smooth algebraic surface $X$ with $X(k) \neq \emptyset$ such that $X(k)$ is known not to be Zariski dense in $X$ ! If one uses nonhomogeneous polynomials, one must instead understand rational points on affine 3 -folds; this seems unlikely to improve the situation. All this suggests that Friedman's question cannot be answered unconditionally without a major advance in arithmetic geometry.

REMARK 1.5. One cannot hope to answer the question using local methods alone. More precisely, if $L$ is any local field of characteristic 0 , and $f(x, y) \in L[x, y]$ is nonconstant, then the induced map $L \times L \rightarrow L$ is not injective. To prove this, choose a point $\left(x_{0}, y_{0}\right) \in L \times L$ where $\partial f / \partial x$ or $\partial f / \partial y$ is nonvanishing, and let $c=f\left(x_{0}, y_{0}\right)$; then the affine curve $f(x, y)=c$ is smooth at $\left(x_{0}, y_{0}\right)$, so by the implicit function theorem it contains infinitely many $L$-points, each of which has the same image under $f$ as $\left(x_{0}, y_{0}\right)$.

REMARK 1.6. If $k$ is any imperfect field, then there exists a polynomial injection $k \times k \rightarrow k$, by a construction that can be found in the proof of Proposition 8 in Cor99. Namely, let $p=\operatorname{char} k$, choose $t \in k-k^{p}$, and use $f(x, y)=x^{p}+t y^{p}$. This applies in particular to any global function field.

REMARK 1.7. The generalized abc-conjecture of [BB94] (more specifically, the 4 -variable analogue) would imply that $f(x, y):=x^{n}+3 y^{n}$ defines a polynomial injection $\mathbb{Q} \times \mathbb{Q} \rightarrow \mathbb{Q}$ for sufficiently large odd integers $n$ : this was observed in [Cor99, Remarque 10].

REMARK 1.8. For the function field $K$ of an irreducible curve over a base field $k$ of characteristic 0 , an analogue of the generalized $a b c$-conjecture is known [Mas86, Lemma 2]. This analogue can be used to show that for some $t \in K$ and $m \geq 1$, the polynomial $f(x, y)=x^{m}+t y^{m}$ defines an injection, under certain technical hypotheses. These hypotheses can be satisfied when $k$ is a number field, for instance. See [Cor99, Proposition 8] for details and for other related results.

2. Proof of theorem. Let $k, F$, and $X$ be as in Theorem 1.1. Let $d=\operatorname{deg} F$. Call a line in $\mathbb{P}^{3}$ trivial if it is given by $x-\zeta z=y-\zeta w=0$ for some $\zeta \in k$ with $\zeta^{d}=1$. Each trivial line is contained in $X$. Let $w$ be the number of roots of 1 in $k$, and let $p$ be a prime number such that $p>3$ and $p \nmid w$. When we speak of the genus of a geometrically irreducible curve, we mean the genus of its smooth projective model. When we say that something holds for "most" elements of $k$ or of $k^{n}$, we mean that it holds outside a thin set in the sense of [Ser97, §9.1]. Such sets arise in the context of the Hilbert irreducibility theorem, which shows that a finite union of thin sets cannot cover all of $k^{n}$. 
Lemma 2.1. Fix an integral closed subscheme $Z$ of $X$. For most $\left(\begin{array}{ll}a & b \\ c & d\end{array}\right) \in$ $\mathrm{GL}_{2}(k) \subset k^{4}$, the inverse image $Y$ of $Z$ under the finite morphism

$\mathbb{P}^{3} \rightarrow \mathbb{P}^{3}, \quad(x: y: z: w) \mapsto\left(a x^{p}+b y^{p}: c x^{p}+d y^{p}: a z^{p}+b w^{p}: c z^{p}+d w^{p}\right)$, satisfies:

(i) If $\operatorname{dim} Z=0$, then $Y(k)=\emptyset$.

(ii) If $Z$ is a trivial line, then $Y(k)$ is contained in a trivial line.

(iii) If $Z$ is any other curve in $X$, then $Y(k)$ is finite.

Proof. We can compute $Y$ in stages, by first taking the forward image of $Z$ under the automorphism

$$
\mathbb{P}^{3} \stackrel{\alpha}{\rightarrow} \mathbb{P}^{3}, \quad(x: y: z: w) \mapsto(a x+b y: c x+d y: a z+b w: c z+d w)
$$

(technically, $\left(\begin{array}{ll}a & b \\ c & d\end{array}\right)$ here should be the inverse of what it was before, but this does not matter), and then pulling back by

$$
\mathbb{P}^{3} \stackrel{\beta}{\rightarrow} \mathbb{P}^{3}, \quad(x: y: z: w) \mapsto\left(x^{p}: y^{p}: z^{p}: w^{p}\right) .
$$

(i) Here $\operatorname{dim} Z=0$. If $Z$ is not a $k$-rational point, then $Z(k)=\emptyset$, so $Y(k)=\emptyset$. If $Z$ is a $k$-rational point, then for most $\left(\begin{array}{ll}a & b \\ c & d\end{array}\right)$ the value of $(a x+b y) /(c x+d y)$ on $Z$ is not a $p$ th power in $k$, so $Y(k)=\emptyset$.

(ii) Here $Z$ is $x-\zeta z=y-\zeta w=0$ for some $\zeta \in k$ with $\zeta^{d}=1$. Then $\alpha(Z)=Z$, so $Y$ is $x^{p}-\zeta z^{p}=y^{p}-\zeta w^{p}=0$. By choice of $p$, the $p$ th-power map on $k$ is injective, and moreover $\zeta=\eta^{p}$ for some $\eta \in k$ with $\eta^{d}=1$. So all points in $Y(k)$ satisfy $x-\eta z=y-\eta w=0$.

(iii) Here $Z$ is an irreducible curve in $X$ that is not a trivial line. If $Z$ is geometrically reducible, then $Z(k)$ is not Zariski dense in $Z$, so $Z(k)$ is finite, and $Y(k)$ is finite too. So assume that $Z$ is geometrically irreducible.

If $y=0$ on $Z$ or if $x / y$ defines a constant rational function on $Z$, then as in (i), for most $\left(\begin{array}{ll}a & b \\ c & d\end{array}\right)$ the value of $x / y$ on $\alpha(Z)$ is not a $p$ th power in $k$, so $Y$ has no $k$-rational points except possibly those where $x=y=0$, so $Y(k)$ is finite.

Suppose that $x / y$ defines a rational function of degree $m>1$ on $Z$. By Bertini's theorem ([Har77, Corollary III.10.9]), $a x+b y$ has distinct zeros on the normalization $Z^{\prime}$ of $Z$, outside the base locus of the linear system given by $\langle x, y\rangle$, for most $a$ and $b$. The same applies to $c x+d y$, so for most $\left(\begin{array}{ll}a & b \\ c & d\end{array}\right)$, the rational function $(a x+b y) /(c x+d y)$ on $Z^{\prime}$ has $m$ simple zeros and $m$ simple poles on $Z^{\prime}$. Adjoining the $p$ th root of this function to the function field of $Z^{\prime}$ yields the function field of a geometrically irreducible curve $C$ of genus greater than 1, by the Hurwitz formula. By [Fal83], $C(k)$ is finite. Since $Y$ admits a dominant rational map to $C$, the set $Y(k)$ is finite too.

Thus we may assume that $x / y$ is of degree 1 on $Z$; in particular, $Z$ is a rational curve. Similarly, we may assume that $z / w$ is of degree 1 on $Z$. If the rational functions $x / y$ and $z / w$ on $Z$ were different, then for most $\left(\begin{array}{ll}a & b \\ c & d\end{array}\right)$, the 
supports of the divisors of $(a x+b y) /(c x+d y)$ and $(a z+b w) /(c z+d w)$ on the normalization of $Z$ would not coincide. Adjoining the $p$ th roots of these functions would lead to a geometrically irreducible curve of genus greater than 1, by the Hurwitz formula again. So $Y(k)$ would be finite as before.

Thus we may assume that $x / y=z / w$ as rational functions on $Z$. So on $Z$, we have

$$
x^{d} F(x, y)=x^{d} F(z, w)=F(x z, x w)=F(x z, y z)=z^{d} F(x, y) .
$$

But $F(x, y)$ does not vanish on $Z$ (since $x / y$ is nonconstant), so $x^{d}-z^{d}$ vanishes on $Z$. Since $Z$ is geometrically irreducible, $x-\zeta z$ vanishes on $Z$ for some $\zeta \in k$ with $\zeta^{d}=1$. But $x / y=z / w$ on $Z$, so $y-\zeta w$ vanishes on $Z$ too. Thus $Z$ is a trivial line, a contradiction.

Let $W$ be the Zariski closure of $X(k)$. By assumption, $\operatorname{dim} W \leq 1$. Applying Lemma 2.1 to each irreducible component of $W$ shows that by replacing $F(x, y)$ with $F\left(a x^{p}+b y^{p}, c x^{p}+d y^{p}\right)$ for suitable $\left(\begin{array}{ll}a & b \\ c & d\end{array}\right)$, we may reduce to the case that $W(k)$ contains at most finitely many points outside the trivial lines. Repeating this construction lets us reduce to the case that $W(k)$ is contained in the trivial lines.

Lemma 2.2. For $G(x, y):=F\left(x^{p}+1, y^{p}+1\right)$ for the new $F$ as above, the equation $G(x, y)=G(z, w)$ has only finitely many solutions over $k$ with $(x, y) \neq(z, w)$.

Proof. Suppose that $x, y, z, w \in k$ are such that $G(x, y)=G(z, w)$. Then there exists $\zeta \in k$ with $\zeta^{d}=1$ such that $x^{p}+1=\zeta\left(z^{p}+1\right)$ and $y^{p}+1=$ $\zeta\left(w^{p}+1\right)$. If $\zeta=1$, then this implies $(x, y)=(z, w)$, by the choice of $p$. If $\zeta \neq 1$, then $x^{p}+1=\zeta\left(z^{p}+1\right)$ defines a geometrically irreducible curve whose projective closure is smooth, and hence of genus $p(p-1) / 2>1$, so by Fal83 it has at most finitely many solutions in $k$. The same applies to $y^{p}+1=\zeta\left(w^{p}+1\right)$, and there are only finitely many $\zeta$.

Lemma 2.3. If $G(x, y) \in k[x, y]$ is such that the equation $G(x, y)=$ $G(z, w)$ has only finitely many solutions over $k$ with $(x, y) \neq(z, w)$, then for most $(a, b) \in k^{2}$ the polynomial $f(x, y):=G\left(a x^{p}+b, a y^{p}+b\right)$ defines an injection $k \times k \rightarrow k$.

Proof. By choice of $p$, if $a \neq 0$ and $b$ is arbitrary, the polynomial $a x^{p}+b$ defines an injection $k \rightarrow k$. Also, for most $(a, b)$, the coordinates of the finitely many exceptional solutions to $G(x, y)=G(z, w)$ are not in the range of this injection.

Theorem 1.1 follows from Lemmas 2.2 and 2.3 ,

Acknowledgements. The idea for this article arose during a discussion session at the Hausdorff Institute in Bonn. I thank Burt Totaro for a 
comment on a first draft of this article, and I thank the referee for a few suggestions.

This research was supported by NSF grant DMS-0841321.

\section{References}

[BB94] J. Browkin and J. Brzeziński, Some remarks on the abc-conjecture, Math. Comp. 62 (1994), no. 206, 931-939.

[Cor99] G. Cornelissen, Stockage diophantien et hypothèse abc généralisée, C. R. Acad. Sci. Paris Sér. I Math. 328 (1999), 3-8.

[Fal83] G. Faltings, Endlichkeitssätze für abelsche Varietäten über Zahlkörpern, Invent. Math. 73 (1983), 349-366, erratum, ibid. 75 (1984), 381; English transl.: Finiteness theorems for abelian varieties over number fields, in: Arithmetic Geometry (Storrs, CT, 1984), Springer, New York, 1986, 9-27.

[Har77] R. Hartshorne, Algebraic Geometry, Grad. Texts in Math. 52, Springer, New York, 1977.

[Mas86] R. C. Mason, Norm form equations. I, J. Number Theory 22 (1986), 190-207.

[Ser97] J.-P. Serre, Lectures on the Mordell-Weil Theorem, 3rd ed., Aspects Math., Vieweg, Braunschweig, 1997.

Bjorn Poonen

Department of Mathematics

Massachusetts Institute of Technology

Cambridge, MA 02139-4307, U.S.A.

E-mail: poonen@math.mit.edu

http://math.mit.edu/ poonen

Received on 25.5.2009

and in revised form on 17.5.2010 